\title{
Combinations provide sustained benefit
}

Immune-checkpoint inhibitors (ICIs) have dramatically altered the prognosis of patients with advanced-stage melanoma. Now, 5-year follow-up data from CheckMate 067 confirm the long-term survival of patients receiving these therapies.

A total of 945 patients were randomized (1:1:1) to receive either nivolumab plus 4 doses of ipilimumab, followed by nivolumab monotherapy; nivolumab monotherapy; or ipilimumab monotherapy until disease progression, unacceptable toxicities, or withdrawal of consent. Progression-free survival (PFS) and overall survival (OS) were the primary end points of this trial.

At a follow-up duration of $\geq 60$ months, median OS durations were not reached, 36.9 and 19.9 months in the nivolumab plus ipilimumab, nivolumab, and ipilimumab groups, respectively. Just over half (52\%) of patients receiving nivolumab plus ipilimumab were alive at 5 years of follow-up monitoring.

A total of 36 patients were continuing to receive treatment at the current data-cut-off. Assessments of treatment-free interval, excluding patients who discontinued follow-up or died prior to subsequent therapy, revealed a median duration of $18.1,1.8$ and 1.9 months in patients in the nivolumab plus ipilimumab, nivolumab and ipilimumab monotherapy groups, respectively.

No notable new adverse events emerged at this data cut-off: $58 \%$ of patients receiving nivolumab plus ipilimumab had grade 3-4 adverse events, compared with $23 \%$ and $28 \%$ in the nivolumab and ipilimumab groups, respectively. The majority of adverse events had resolved at the time of data cut-off, with the exception of endocrine events, including 6 patients with grade $3-4$ diabetes, and 68 patients with thyroid disorders of any grade.

These data confirm that the benefits of ICIs can be sustained for $>5$ years in patients with advanced-stage melanoma who respond to these agents and that the majority of adverse events are reversible. However, irreversible adverse events can occur and will likely require lifelong management.

Peter Sidaway

ORIGINAL ARTICLE Larkin, J. et al. Five-year survival with combined nivolumab and ipilimumab in advanced melanoma. N. Engl.J. Med. https://doi.org/10.1056/NEJMoa1910836 (2019)

\section{GASTROINTESTINAL CANCER}

\section{Nivolumab effective in oesophageal carcinoma}

Patients with recurrent oesophageal squamous cell carcinoma (ESCC) after frontline fluoropyrimidine and platinum-based combinations generally receive docetaxel or paclitaxel, which are associated with poor long-term survival. According to data from the phase III ATTRACTION-3 trial, reported at the ESMO Congress 2019 and in the Lancet Oncology, nivolumab is a promising agent for these patients.

In this trial, patients with ESCC (including

adenosquamous carcinomas) were randomly assigned to receive either nivolumab $(n=210)$ or standard second-line chemotherapy $(n=209)$. At 17.6 months, the median overall survival (OS) durations were 10.9 months and 8.4 months, respectively (HR 0.77; $P=0.02$ ). For patients receiving nivolumab, the median $O S$ duration was the same regardless of the level of tumour expression of PD-L1 ( $<1 \%$ or $\geq 1 \%$ ). With chemotherapy, median OS durations were 9.3 months for patients harbouring tumours with $<1 \%$ PD-L1 expression and 8.1 months for those with $\geq 1 \%$ PD-L1.

The objective response rate was $19 \%$ with nivolumab and $22 \%$ with chemotherapy. Overall, nivolumab was associated with inferior progression-free survival (median 1.7 months versus 3.4 months with chemotherapy; HR 1.08), but a superior median duration of response (6.9 months versus 3.9 months).

The incidence of grade $\geq 3$ treatment-related adverse events was $18 \%$ with nivolumab and $64 \%$ with chemotherapy; two fatalities in the nivolumab group and three with chemotherapy were deemed to be treatment-related. Healthrelated quality of life was assessed with a threelevel questionnaire (EQ-5D-3L), with results favouring nivolumab over chemotherapy.

In July 2019, the FDA approved pembrolizumab for patients with advanced-stage ESCC expressing PD-L1 (combined positive score (CPS) $\geq 10$ ) in the second-line setting and beyond. This approval was based on the OS results of the KEYNOTE-181 trial: median 10.3 months with pembrolizumab versus 6.7 months with chemotherapy (HR 0.64). Whether nivolumab will be approved for this indication irrespective of PD-L1 expression remains to be seen.

Diana Romero

ORIGINAL ARTICLE Kato, K et al Lancet Oncol. https:// doi.org/10.1016/S1470-2045(19)30626-6 (2019)

\section{BEACON of hope in $B R A F^{\mathrm{V} 600 \mathrm{E}} \mathrm{CRC}$}

BRAF mutations are detected in up to $20 \%$ of patients with metastatic colorectal cancer ( $\mathrm{mCRC}$ ) and portend a very poor prognosis. Now, data from the phase III BEACON CRC trial, recently reported at the ESMO Congress 2019 and in the New England Journal of Medicine, demonstrate the promising efficacy of a rational combination of targeted agents.

"BRAF inhibitors as monotherapy have failed to improve the outcomes of patients with $B R A F^{\mathrm{V} 600 \mathrm{E}}$-mutated $\mathrm{mCRC}$," states corresponding author Josep Tabernero. This finding is contrary to the results obtained in other $B R A F^{\mathrm{V} 600}$-mutated cancers, particularly melanoma, and is explained by rapid activation of bypass signalling via EGFR in $\mathrm{mCRC}$.

"Thus, we sought to potentiate BRAF-targeted therapy with an EGFR inhibitor, with or without a MEK inhibitor," explains Tabernero.

Accordingly, 665 patients with previously treated $B R A F^{\mathrm{V} 600 \mathrm{E}}$-mutated $\mathrm{mCRC}$ were randomly assigned (1:1:1) to either triplet therapy with encorafenib, binimetinib and cetuximab, doublet therapy with encorafenib and cetuximab, or control treatment with cetuximab and irinotecan-based chemotherapy. The median overall survival durations were 9.0 months, 8.4 months and 5.4 months, respectively (HR 0.52 for the triplet and 0.60 for the doublet versus the control treatment; $P<0.001$ for both comparisons). The objective response rates were $26 \%, 20 \%$ and $2 \%$, respectively. Importantly, the novel combinations had favourable toxicity profiles, with a lower rate of grade $\geq 3$ adverse events with the triplet $(58 \%)$ and with the doublet (50\%) than with control treatment (61\%).

"Our findings point to a paradigm shift in the treatment of BRAF-mutant $\mathrm{mCRC}$ and warrant further investigation to better define the benefits of both the triplet and doublet therapies," opines Tabernero. Indeed, the study was not powered to compare the triplet and doublet therapies; for some patients the doublet might be sufficient. "These novel combinations are certainly showing promise in reverting the resistance of this disease to therapy and must be pursued swiftly," concludes Tabernero. Notably, the ongoing ANCHOR-CRC trial will provide insights into the efficacy of the triplet in patients with treatment-naive $\mathrm{mCRC}$.

David Killock

ORIGINAL ARTICLE Kopetz, S. et al. Encorafenib, binimetinib, and cetuximab in BRAF V600E-mutated colorectal cancer. N. Engl.J. Med. https://doi.org/10.1056/NEJMoa1908075 (2019) 